\section{Fatores médicos e não-médicos associados às taxas de cesariana em um hospital universitário no Sul do Brasil}

\author{
Medical and non-medical factors associated with \\ cesarean section rates in a university hospital in \\ southern Brazil
}

Paulo Fontoura Freitas 1,2,3

Thiago Mamôru Sakae 1,2

Maria Eduarda M. Lebarbechon Polli Jacomino ${ }^{3}$

\footnotetext{
1 Departamento de Saúde Pública, Universidade

Federal de Santa Catarina, Florianópolis, Brasil. 2 Universidade do Sul de Santa Catarina, Tubarão, Brasil.

3 Hospital Universitário Universidade Federal de Santa Catarina, Florianópolis, Brasil.

Correspondencia P. F. Freitas

Departamento de Saúde Pública, Universidade Federal de Santa Catarina. Beco da Lua 232, Florianópolis, SC 88062-475, Brasil. preitas@ccs.ufsc.br
}

\begin{abstract}
The objective of this study was to describe factors associated with the increase in cesarean rates in a university hospital in 2002 and 2004, exploring medical and non-medical factors. A crosssectional study investigated 2,905 deliveries: 1,441 in 2002 and 1,464 in 2004. Differences in adjusted prevalence rates using Poisson regression and attributable risk percent were estimated for the associations between cesarean section and demographic, clinical, reproductive, institutional, obstetric, and delivery-related factors. The cesarean rate increased from $28.4 \%$ in 2002 to $36.7 \%$ in 2004. Higher maternal schooling, time of day at delivery, illness during pregnancy, and number of prenatal visits were associated with the excess rate in 2004 compared to 2002. The increased cesarean rate can be attributed at least partially to an increase in relative clinical indications and non-medical factors.
\end{abstract}

Cesarean Section; Risk Factors; Attributable Risk; University Hospitals
Introdução

O aprimoramento das técnicas cirúrgicas e o uso de antibióticos contribuíram para tornar a cesariana um método relativamente prático e seguro, levando à disseminação do caráter aparentemente inócuo de sua prática, sobretudo por meio da mídia, com supervalorização da tecnologia 1,2 .

Como resultado, vem ocorrendo em todo o mundo um grande alargamento das indicações médicas de cesariana e, simultaneamente, um expressivo crescimento de outras indicações para este tipo de parto ${ }^{3}$.

Em 1985, a Organização Mundial da Saúde (OMS) mostrou que uma taxa de cesarianas maior do que $15 \%$ é medicamente injustificável 4 . Entretanto, um aumento global das taxas de cesarianas vem ocorrendo. O Brasil apresenta uma das taxas mais elevadas do mundo 5,6.

Estudo recente 6 mostrou que doze entre dezenove países latino-americanos estudados apresentavam taxas de cesariana acima do proposto pela OMS, variando entre 16,8\% (Colôm bia) e $40 \%$ (Chile). No mesmo estudo, o Brasil ocupava a segunda posição, com $27,1 \%$. Dados do Departamento de Informática do SUS (DATASUS) apontaram taxas de cesariana crescentes desde o ano de 1994, 32\%, chegando a $40,2 \%$ em 1996. Após esse período, houve redução até 1999, quando as taxas foram de 36,9\%, aumentando novamente para 39,9\% em de 20027. 
Acompanhando um aumento das taxas no Brasil, Santa Catarina possuía em 2002 o oitavo maior porcentual de cesarianas entre os estados, com valores de 43,8\% 8. Em 2004, as cesarianas já eram responsáveis por $48,3 \%$ do total de partos no estado 7 . No mesmo ano, Florianópolis apresentou taxas de cesariana em torno de $50 \% 7$.

Na maternidade do Hospital Universitário da Universidade Federal de Santa Catarina (HU-UFSC), as taxas também vêm aumentando nos últimos anos com um crescimento de aproximadamente 8\% nas taxas de 1996 a 2004 (Serviço de Enfermagem do Centro Obstétrico do HUUSFC. Livro de Registro de Partos, 1995 a 2005).

Profissionais e movimentos sociais engajados em práticas e valores com a noção da humanização da assistência ao parto e ao nascimento têm se mostrado preocupados com o crescente aumento das taxas de cesariana 9. Principalmente porque, apesar de indicadas sob condições médicas estritas, o abuso das cesarianas está relacionado a complicações maternas e perinatais, bem como gastos desnecessários ao sistema de saúde 8,10 . Risco de prematuridade, em decorrência do uso não criterioso da tecnologia, como o ultra-som, falhas na determinação precisa da idade gestacional e associadas com cesarianas decididas eletivamente têm sido sugeridos desde os anos 80, no Brasil 5.

Segundo Diniz 11, as diretrizes da Rede de Humanização do Parto e do Nascimento sintetizam um conjunto importante de pesquisas sobre parto no mundo inteiro e propõem uma crítica à excessiva medicalização do parto, orientado pelo modelo tecnocrático de assistência.

A busca por fatores que justifiquem esses aumentos é essencial para que soluções possam ser pensadas. Estudos nacionais e internacionais mostram que as indicações absolutas, em que a vida da mãe e/ou do feto estejam em risco, vêm sendo superadas por indicações relativas e até mesmo as não-médicas 12,13.

Recentemente Bailit et al. 13, na Carolina do Norte, Estados Unidos, ao encontrarem taxas crescentes de cesariana concomitantes a uma diminuição no risco perinatal concluíram que a justificativa para o crescimento possa estar associada unicamente a alterações nas características maternas e mudanças nas condutas médica e hospitalar 13,14.

Por outro lado, variações nas taxas de acordo com a região apontam para uma correlação positiva das taxas com desenvolvimento sócioeconômico, com taxas médias de cesariana menores para os municípios socioeconomicamente menos favorecidos 2 .

Freitas et al. 15 sugerem que fatores sócioeconômicos, de idade, escolaridade e etnia re- lacionados à chance de cesariana, em grupos nos quais são esperadas melhores condições de saúde materna e menor risco obstétrico, estejam relacionados ao abuso da tecnologia médica no atendimento ao parto.

Segundo Faúndes \& Cecatti ${ }^{8}$, a organização atual da atenção obstétrica tem importante papel nas elevadas taxas de cesariana. Os autores destacam o papel da conveniência de uma intervenção programada para o obstetra, a incerteza quanto à possibilidade de hipóxia ou trauma fetal e a falta de preparo da mulher para o parto como os principais fatores ligados à preferência médica na ocorrência de cesarianas 8 .

Em recente estudo 16, realizado em Ribeirão Preto, São Paulo, foi observado um aumento nas taxas de cesariana entre as mulheres de maior nível sócio-econômico e, conseqüentemente, de menor risco obstétrico, fato também encontrado no Sul do Brasil 15. No estudo de Ribeirão Preto 16 , chamou a atenção o fato de que nas categorias particulares e de pré-pagamento não foram encontrados diagnósticos que justificassem a decisão pela cesariana, baseada em indicações médicas.

Dessa forma, diferentes estudos vêm sugerindo que: fatores pessoais, da mulher e de seu ambiente social 3,5, bem como regionais e institucionais relacionados à organização dos serviços 13 constituem aspectos não-médicos relacionados ao parto no Brasil, influenciando a cultura da prática obstétrica vigente.

A investigação dos fatores associados ao aumento das taxas no HU-UFSC no período definido pelo presente estudo busca colaborar no entendimento mais amplo da questão. Os resultados aqui apresentados visam a subsidiar o planejamento de ações dirigidas ao problema.

\section{Métodos}

\section{Delineamento}

Um estudo com delineamento transversal utilizou dados coletados e registrados rotineiramente como parte da admissão, pré-parto e parto na maternidade do HU-UFSC.

\section{Local do estudo}

O HU-UFSC tem atendimento público e gratuito sendo reconhecido como Hospital Amigo da Criança. Pela iniciativa de um grupo de profissionais trabalhando desde 1988 na filosofia do atendimento humanizado ao parto, o projeto que garante às mulheres a presença de um acompanhante durante o parto ("Lei do Acom- 
panhamento") foi aprovada em nível estadual em 2005.

\section{Fonte de coleta dos dados}

Os dados analisados foram obtidos utilizandose o protocolo da História Clínica Perinatal Base (HCPB), instrumento desenvolvido pelo Centro Latino-Americano de Perinatologia (CLAP; http://www.clap.ops-oms.org/) e utilizado desde 1995 na maternidade do HU-UFSC. A informação no protocolo HCPB é revisada e validada rotineiramente tendo como base os prontuários clínicos antes de ser armazenada usando-se o programa do Sistema Informático Perinatal do CLAP (SIP; http://www.clap.ops-oms.org/web_ 2005/SIP2007/sip2007.htm). No estudo, os dados digitados no SIP foram exportados para o programa Stata 8.0 (Stata Corp., College Station, Estados Unidos) no qual foram conduzidas as análises.

\section{Categorias e definição das variáveis}

Os fatores investigados como potencialmente associados ao tipo de parto foram categorizados em três grupos: (1) sócio-demográficos (idade materna [ $<20$ anos, 20-30 anos, $>35$ anos] e escolaridade [nenhuma/fundamental, médio/superior]); (2) reprodutivos e clínicos (cesárea prévia [sim, não], paridade [primípara, multípara], idade gestacional [até 36, 37-40, 41 semanas ou mais], número de consultas pré-natal [até 6,7 ou mais] e patologia na gestação [sim/não]); e (3) do parto, institucionais e/ou da prática obstétrica (ocitocina [uso/não-uso], turno do parto [19:00 até 6:59 e 7:00 até 18:59] e apresentação [cefálica, não cefálica]).

Uma vez que o registro da variável "patologia”, para o ano de 2002, não permitia a diferenciação entre co-morbidades específicas, optou-se pela classificação "presença de qualquer patologia" para os dois anos com a finalidade de obter comparabilidade.

\section{Processamento e análise estatística dos} dados

A informação foi obtida para $97,2 \%$ dos partos ocorridos em 2002 (1.441) e 96,5\% em 2004 (1.464). Foram excluídas 43 mulheres para as quais a variável tipo de parto não estava disponível, 16 mulheres em 2002 e 27 em 2004. Também, decidiu-se por não incluir os 55 partos registrados como "múltiplos", uma vez que para uma proporção importante destes (42\%) o número de identificação materna não aparecia para nascimentos subseqüentes, colocando em dúvida a veracidade da informação.
Diferenças nas proporções de mulheres de acordo com grupos específicos das variáveis de interesse e ano do parto foram investigadas e testadas utilizando-se o teste do qui-quadrado no nível de significância de 95\% ( $\mathrm{p}<0,05)$.

Razões de prevalência (RPs) de cesariana, brutas e ajustadas, para todas as variáveis nos dois anos foram obtidas utilizando-se análise multivariada com regressão de Poisson, ajustadas pelo método de variância robusta, o qual mostrou fornecer estimativas corretas, sendo uma melhor alternativa na análise de estudos transversais com desfechos binários, quando comparada à regressão logística 17.

O excesso no risco (RP > 1) foi calculado tendo como categoria de referência os grupos com menor prevalência de cesariana.

O cálculo das RPs ajustadas no estudo se dá de acordo com um modelo teórico que busca organizar as variáveis hierarquicamente de acordo com sua posição, distal ou proximal, em relação ao desfecho (Figura 1). Assim, as variáveis situadas nos níveis mais superiores são tratadas como confundidoras enquanto aquelas situadas nos níveis mais baixos são analisadas como potenciais mediadoras dos efeitos daquelas nos níveis mais distais.

Figura 1

Modelo hierarquizado dos fatores influenciando a ocorrência de cesariana.

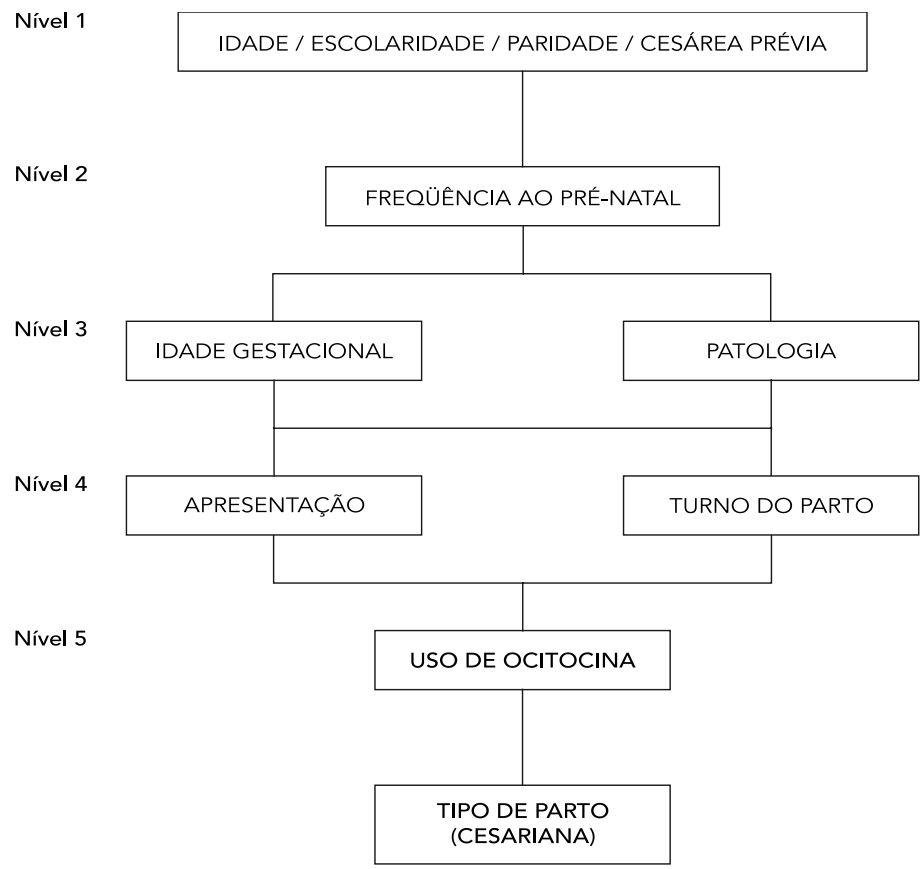


O risco atribuível percentual (RAP) de acordo com o ano do parto e fatores associados à ocorrência de cesariana foi calculado para as categorias apresentando as RPs mais elevadas na análise multivariada. O RAP estima a contribuição específica do grupo de risco no excesso de cesarianas em 2004 quando comparado a 2002 e foi calculado como:

$\mathrm{RAP}=\underline{(\mathrm{RR}-1)}$

$\mathrm{RR}$

Por definição, o RAP expressa a proporção do excesso no risco entre os expostos que pode ser atribuído à exposição, e nos estudos em que uma relação de causalidade pode ser atribuída, entre exposição e desfecho, o RAP poderá ser interpretado como uma "verdadeira fração etiológica". Entretanto, o termo excess fraction, ou seja, excesso como proporção da prevalência total, tem sido sugerido como uma alternativa para o risco atribuível, quando dados de incidência não estejam disponíveis 18 .

\section{Critérios éticos da pesquisa}

O presente estudo foi aprovado pelo Comitê de Ética e Pesquisa com seres humanos da Universidade Federal de Santa Catarina, processo 187/2005, estando de acordo com a Resolução $n^{o}$. 196/96 do Conselho Nacional de Saúde.

\section{Resultados}

Ocorreram 3.003 partos na maternidade do HU-UFSC nos dois anos estudados. A análise se refere à informação obtida para 96,7\% destes, ou seja, 2.905 partos (1.441 em 2002 e 1.464 em 2004).

As taxas de cesariana aumentaram de $28,4 \%$ em 2002 para 36,7\% em 2004.

A distribuição das mulheres nos dois anos de acordo as variáveis sócio-demográficas, reprodutivas, institucionais e com patologia na gestação foram semelhantes, exceto para escolaridade mais elevada, primiparidade e cesárea prévia, cujas proporções foram maiores em 2004 quando comparadas a 2002 (Tabela 1).

Uma maior prevalência de cesarianas foi encontrada para as mulheres tendo parto no ano de 2004 e entre aquelas no grupo de idade e escolaridade mais elevadas (Tabela 2). Após o ajuste para confundimento, de acordo com a hierarquia apresentada na Figura 1, o ano de 2004 e as idades mais avançadas mantiveram o efeito enquanto que escolaridade perdeu efeito e significância estatística. A prevalência de cesarianas foi significativamente maior entre as mulheres de 20-34 e entre aquelas com mais de 35 anos se comparadas àquelas com idade menor que 20 anos, mesmo depois de ajustar para efeito das outras variáveis.

Maiores prevalências de cesariana foram encontradas entre as primíparas; multíparas com cesariana prévia; nas gestações pré ou pós-termo; maior número de consultas pré-natal e nas mulheres com patologia na gestação. Após ajuste para confundimento, todas as variáveis do grupo seguiram apresentando um efeito independente e estatisticamente significante, com exceção de primiparidade. A RP se mostrou especialmente mais elevada para as mulheres com história de cesariana anterior, com um risco quase três vezes maior do que entre multíparas sem esta característica. Para os demais fatores, o excesso no risco esteve entre $10 \%$ e $20 \%$.

Uma maior taxa de cesarianas foi encontrada para os partos ocorridos durante o período diurno, aqueles com apresentação não-cefálica à admissão e em partos em que houve uso de ocitocina. Mesmo depois do ajuste, as RPs seguiram significativamente mais altas nesses grupos. A prevalência de cesariana nos partos com apresentação não-cefálica foi quase três vezes maior do que aqueles com apresentação cefálica. Em relação ao uso de ocitocina, a prevalência foi $60 \%$ maior entre as mulheres que usaram a droga. Já para o turno em que o parto ocorreu, os partos diurnos tiveram prevalências $26 \%$ maiores que os da madrugada.

As prevalências de cesarianas para as categorias apresentando o maior risco para este tipo de parto para todas as variáveis investigadas, bem como o RAP para cada, de acordo com ano do parto, podem ser observadas na Tabela 3 .

Apresentação não-cefálica, cesariana prévia, idade acima dos 35 anos e uso de ocitocina foram responsáveis, em qualquer um dos dois anos, pelas maiores contribuições no excesso de cesarianas. Turno do parto, patologia na gestação e escolaridade foram os fatores que apresentaram os maiores acréscimos na fração do excesso no ano de 2004, se comparado ao ano de 2002. Por outro lado, idade acima dos 35 anos, idade gestacional menor que 37 semanas e apresentação não-cefálica diminuíram sua contribuição relativa no período.

\section{Discussão}

Foram analisados $96,7 \%$ dos partos ocorridos nos dois anos de interesse. As exclusões, incluindo os partos múltiplos responsáveis por $1,7 \%$ dos nascimentos, não afetaram as conclusões relativas aos objetivos centrais do estudo. 
Distribuição das mulheres de acordo com as variáveis sociodemográficas, reprodutivas e institucionais, patologia na gestação e ano do parto na maternidade do Hospital Universitário da Universidade Federal de Santa Catarina. Florianópolis, Santa Catarina, Brasil, nos anos de 2002 e 2004.

\begin{tabular}{|c|c|c|c|c|c|}
\hline \multirow[t]{2}{*}{ Variável } & \multicolumn{2}{|c|}{2002} & \multicolumn{2}{|c|}{2004} & \multirow[t]{2}{*}{ Valor de $p$} \\
\hline & $\mathbf{n}$ & $\%$ & $\mathrm{n}$ & $\%$ & \\
\hline \multicolumn{6}{|l|}{ Idade (anos) } \\
\hline$<20$ & 293 & 20,3 & 283 & 19,3 & \\
\hline $20-34$ & 1.020 & 70,1 & 1.044 & 71,3 & 0,84 \\
\hline 35 e mais & 128 & 8,9 & 137 & 9,4 & 0,53 \\
\hline \multicolumn{6}{|l|}{ Escolaridade } \\
\hline Nenhuma/Fundamental & 763 & 54,7 & 709 & 49,9 & \\
\hline Médio/Superior & 632 & 45,3 & 714 & 50,2 & $<0,01$ \\
\hline \multicolumn{6}{|l|}{ Paridade } \\
\hline Primípara & 508 & 35,4 & 653 & 44,6 & \\
\hline Multípara & 933 & 64,6 & 810 & 55,4 & $<0,001$ \\
\hline \multicolumn{6}{|l|}{ Cesariana prévia } \\
\hline Sim & 160 & 17,1 & 227 & 28,0 & \\
\hline Não & 773 & 82,9 & 584 & 72,0 & $<0,001$ \\
\hline \multicolumn{6}{|l|}{ Idade gestacional (semanas) } \\
\hline $37-41$ e 6 dias & 1.193 & 82,8 & 1.223 & 83,6 & \\
\hline$<37$ & 194 & 13,5 & 179 & 12,2 & 0,17 \\
\hline$\leq 42$ & 54 & 3,7 & 62 & 4,2 & 0,52 \\
\hline \multicolumn{6}{|l|}{ Pré-natal (consultas) } \\
\hline$\leq 6$ & 812 & 56,3 & 811 & 55,4 & \\
\hline$>6$ & 629 & 43,7 & 653 & 44,6 & 0,63 \\
\hline \multicolumn{6}{|l|}{ Patologia } \\
\hline Sim & 477 & 33,1 & 490 & 33,5 & 0,86 \\
\hline Não & 964 & 66,9 & 974 & 66,5 & \\
\hline \multicolumn{6}{|l|}{ Turno } \\
\hline 00:00-06:59 & 337 & 23,4 & 339 & 23,2 & \\
\hline 07:00-23:59 & 1.104 & 76,6 & 1.125 & 76,8 & 0,917 \\
\hline \multicolumn{6}{|l|}{ Apresentação } \\
\hline Cefálica & 1.401 & 97,5 & 1.407 & 96,3 & \\
\hline Outras & 36 & 2,5 & 54 & 3,7 & 0,082 \\
\hline \multicolumn{6}{|l|}{ Ocitocina } \\
\hline Sim & 674 & 46,8 & 693 & 47,3 & \\
\hline Não & 767 & 53,2 & 771 & 52,7 & 0,79 \\
\hline
\end{tabular}

Dados não obtidos para: escolaridade (46 mulheres em 2002 e 41 em 2004); cesárea prévia (8 mulheres em 2002); e patologia (4 mulheres em 2002 e 3 em 2004).

Os resultados mostraram que diferenças no perfil das mulheres nos dois anos aparentemente não poderiam explicar por si só a magnitude dos excessos encontrados nas taxas ao se comparar 2002 e 2004. As diferenças mais importantes se relacionaram com variáveis representando ausência de indicações estritamente médicas, como paridade, ou relacionadas com indicações relativas, como existência de uma cesárea anterior.

Um dos resultados mais importantes do presente estudo foi o achado de que um aumento nas prevalências em 2004 ocorreu independentemente das características maternas sócio-demográficas e reprodutivas, ou seja, diferenças no perfil das parturientes atendidas não explicam este aumento. A maior prevalência de cesarianas encontrada para mulheres acima de 35 anos condiz com achados na literatura 19,20. A hipótese de que pacientes mais velhas teriam mais comorbidades ou estivessem relacionadas a outras características que as levassem a ter mais cesarianas foi levantada por alguns autores 20,21 . No 
Prevalência e razões de prevalência brutas (RP) e ajustadas (RPaj) e intervalo de confiança de 95\% (IC95\%) das cesarianas para variáveis sociodemográficas, clínicas, reprodutivas e institucionais e ano do parto na maternidade do Hospital Universitário da Universidade Federal de Santa Catarina, Florianópolis. Santa Catarina, Brasil, nos anos de 2002 e 2004.

\begin{tabular}{|c|c|c|c|c|c|}
\hline Variáveis independentes & $\mathrm{n}$ & $\%$ & RP & RPaj & IC95\% \\
\hline \multicolumn{6}{|l|}{ Ano } \\
\hline 2002 & 404 & 28,4 & Referência & Referência & \\
\hline 2004 & 528 & 36,7 & 1,29 & 1,22 & $1,10-1,36$ \\
\hline \multicolumn{6}{|l|}{ Idade (anos) * } \\
\hline$<20$ & 140 & 24,6 & Referência & & \\
\hline $20-34$ & 681 & 33,0 & 1,3 & 1,44 & $1,18-1,75$ \\
\hline 35 e mais & 118 & 44,7 & 1,69 & 1,87 & $1,43-2,46$ \\
\hline \multicolumn{6}{|l|}{ Escolaridade * } \\
\hline$\leq$ Fundamental & 450 & 31,4 & Referência & Referência & \\
\hline$\geq$ Médio & 456 & 34,0 & 1,08 & 0,98 & $0,88-1,09$ \\
\hline \multicolumn{6}{|l|}{ Paridade * } \\
\hline Multípara & 534 & 31,3 & Referência & & \\
\hline Primípara & 397 & 34,6 & 1,1 & 1,12 & $1,00-1,26$ \\
\hline \multicolumn{6}{|l|}{ Cesária prévia * } \\
\hline Não & 292 & 22,0 & Referência & & \\
\hline $\operatorname{Sim}$ & 242 & 63,4 & 2,88 & 2,64 & $2,31-3,00$ \\
\hline \multicolumn{6}{|c|}{ Idade gestacional (semanas) ** } \\
\hline $37-41$ e 6 dias & 759 & 31,2 & Referência & & \\
\hline$<37$ & 145 & 39,0 & 1,25 & 1,27 & $1,05-1,54$ \\
\hline$\geq 42$ & 46 & 40,0 & 1,28 & 1,23 & $0,91-1,67$ \\
\hline \multicolumn{6}{|l|}{ Pré-natal (consultas) * } \\
\hline$\leq 6$ & 482 & 30,3 & Referência & & \\
\hline$\geq 7$ & 450 & 35,4 & 1,16 & 1,14 & $1,03-1,27$ \\
\hline \multicolumn{6}{|l|}{ Patologia ** } \\
\hline Não & 568 & 29,8 & Referência & & \\
\hline $\operatorname{Sim}$ & 354 & 38,0 & 1,27 & 1,23 & $1,11-1,36$ \\
\hline \multicolumn{6}{|l|}{ 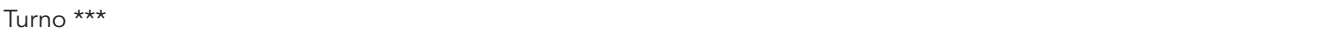 } \\
\hline 00:00-06:59 & 167 & 25,3 & Referência & & \\
\hline 07:00-23:59 & 765 & 34,8 & 1,37 & 1,26 & $1,10-1,45$ \\
\hline \multicolumn{6}{|l|}{ Apresentação *** } \\
\hline Cefálica & 846 & 30,6 & Referência & & \\
\hline Outras & 79 & 87,8 & 2,86 & 2,84 & $2,55-3,16$ \\
\hline \multicolumn{6}{|l|}{ Ocitocina \# } \\
\hline Não & 376 & 24,9 & Referência & & \\
\hline Sim & 556 & 41,2 & 1,65 & 1,56 & $1,03-1,26$ \\
\hline
\end{tabular}

Ajuste para: * todas as outras variáveis no Nível 1 e ano do parto; ** nos Níveis 1 e 2, outras variáveis no mesmo nível e ano do parto; ${ }^{\star \star \star}$ nos Níveis 1, 2 e 3, outras variáveis no mesmo nível e ano do parto; \# nos Níveis 1, 2, 3 e 4 e ano do parto.

entanto, no presente estudo, concordando com outro recente 22 , essa categoria ainda mostrou grande associação com cesarianas após ajuste para confundimento, não havendo justificativa para maior prevalência nesse grupo, de acordo com as variáveis estudadas.

A maior prevalência de cesarianas entre primíparas tem sido descrita como inapropriada para justificar a operação 14,23 . Nesse sentido, o entendimento dos fatores associados com uma maior taxa entre esse grupo de mulheres deve ser especialmente investigado, principalmente porque a existência de história de cesariana prévia carrega riscos acumulados de um novo parto cirúrgico.

Ainda que no presente estudo o número de cesarianas anteriores não tenha sido avaliado, o ditado "uma vez cesariana, sempre cesariana" 
Prevalência de cesariana e risco atribuível percentual (RAP) para as categorias com maior risco de cesariana na maternidade do Hospital Universitário da Universidade Federal de Santa Catarina. Florianópolis, Santa Catarina, Brasil, nos anos de 2002 e 2004 de acordo com ano do parto.

\begin{tabular}{|c|c|c|c|c|c|c|}
\hline \multirow[t]{2}{*}{ Variável } & \multicolumn{3}{|c|}{2002} & \multicolumn{3}{|c|}{2004} \\
\hline & $\mathbf{n}$ & $\%$ & RAP & $\mathbf{n}$ & $\%$ & RAP \\
\hline Idade: $>35$ anos & 100 & 35,5 & 46,52 & 141 & 47,8 & 42,65 \\
\hline Escolaridade: Médio/Superior & 182 & 29,0 & 3,51 & 268 & 38,5 & 8,73 \\
\hline Paridade: Primípara & 148 & 29,4 & 5,22 & 249 & 38,5 & 8,70 \\
\hline Cesariana anterior: Sim & 96 & 60,0 & 65,0 & 146 & 65,8 & 64,4 \\
\hline Idade gestacional: $<37$ semanas & 183 & 33,8 & 29,87 & 227 & 42,4 & 21,35 \\
\hline Pré-natal: $\geq 7$ consultas & 189 & 30,3 & 11,63 & 261 & 40,2 & 15,75 \\
\hline Apresentação: não-cefálica & 30 & 83,3 & 67,00 & 49 & 90,7 & 61,99 \\
\hline Ocitocina: Sim & 238 & 35,7 & 38,80 & 318 & 46,4 & 39,68 \\
\hline Patologia: Sim & 152 & 32,3 & 18,15 & 212 & 43,6 & 23,82 \\
\hline Turno: 07:00-23:59 & 327 & 30,0 & 23,08 & 438 & 39,5 & 30,25 \\
\hline
\end{tabular}

pode ser aqui aplicado. No entanto, não há evidência médica de um aumento no risco associado com parto vaginal entre mulheres com cesariana prévia 24,25 .

Partos a termo mostraram menor risco de cesariana quando comparados aos pré e póstermo. Esse achado é bem descrito na literatura, principalmente no relativo aos pós-termo $26,27,28$, categoria aqui não analisada individualmente. Um maior risco de cesariana entre as mulheres de idade gestacional pré-termo continuou existindo após o ajuste, e outros fatores que não a coexistência de patologias estariam provavelmente explicando este excesso.

Uma associação entre aumento nas taxas e maior freqüência ao pré-natal se manteve após ajuste para as outras variáveis, trazendo novamente à tona a influência de fatores não-médicos. Maiores taxas entre as mulheres com sete ou mais consultas pré-natal também foram descritas por Gomes et al. 19. Conforme já levantado por Freitas et al. 15, aqui também poder-se-ia pensar que um maior contato com o obstetra possa ter influenciado a decisão por cesariana. No entanto, a maternidade do HU-UFSC funciona com escala de plantões em atendimento de emergência e o acompanhamento pré-natal dentro do hospital é realizado por acadêmicos sob supervisão de médicos-professores, colocando em dúvida a influência por este caminho. Além do mais, os dados colhidos não trazem a informação sobre o responsável ou o local do pré-natal.

É importante ter em mente que os resultados aqui encontrados devem ser interpretados levando-se em conta as características e os limites permitidos pelas variáveis estudadas. Para algumas delas, um número de fatores não considerados na presente análise pode operar durante o pré-natal e parto influenciando a chance de cesariana, por exemplo, intercorrências durante gestação levando a um início mais precoce e/ou um maior número de consultas no pré-natal.

Ainda que resultados de um aumento na ocorrência de parto cesáreo entre as mulheres com história de patologia na gestação estivesse dentro do esperado, heterogeneidade na definição desta variável nos dois anos impossibilita maiores conclusões. Apesar do estudo não ter investigado patologia específica, este conceito já está bem consolidado na prática obstétrica 27,28,29, em particular a associação com diabetes (prévia ou gestacional) 14,29,30.

Outro achado de consistência na literatura é a ocorrência de cesariana em grande parte dos partos cuja apresentação não é cefálica ${ }^{26}$. No presente estudo, um número de partos registrados pelo obstetra como apresentação não-cefálica, bem acima do esperado, pode ser atribuído à necessidade de justificar cesarianas sem indicação médica, utilizando tal indicação tida pela comunidade obstétrica como absoluta. Em um estudo realizado no Rio de Janeiro ${ }^{2}$, entrevistas com obstetras mostraram que o ensino e a prática de partos vaginais nesses casos estão desaparecendo, o que contribui para a insegurança do médico frente às inúmeras variações que podem ocorrer durante o trabalho de parto. Nesse contexto, estão as "cesarianas profiláticas” ou defensivas quanto a questões éticas e judiciais em 
casos em que o parto vaginal aponta para complicações 2. Paralelo a isso, o aperfeiçoamento da técnica cirúrgica e anestésica também contribuem para a opção pela cesariana ${ }^{9}$.

A prática médica é novamente questionada quando abordamos resultados referentes ao maior risco de cesariana entre partos seguindo condições explicitamente não-médicas, como hora do parto e uso de ocitocina. Indução do parto levando à cesariana tem sido citada na literatura 26. Embora descrita como associada à indução em nulíparas, aqui o efeito continuou significante após ajuste para paridade.

Quanto à relatada ocorrência de cesariana bem mais alta em determinados períodos do dia, outros estudos levantando o fator comentam a óbvia inexistência de justificativas médicas para este fenômeno ${ }^{19}$. Isso reforça a idéia de que os fatores relacionados à prática médica têm grande influência na decisão pela cesariana. Como já sugerido, para outros autores parece haver uma adequação dos horários do obstetra em sua agenda privada 8 .

Os resultados aqui apresentados sugerem que fatores relacionados com indicações relativas e não-médicas teriam sido responsáveis por grande parte do excesso ao se comparar as taxas de cesariana em 2002 e 2004 . História prévia de cesariana e apresentação não-cefálica, embora não tenham perdido a magnitude ao se comparar os dois anos, tiveram sua contribuição reduzida quanto ao excesso nas taxas para o ano de 2004, dando subsidio à hipótese de que razões puramente médicas não justificam o aumento encontrado na prevalência de cesariana.

Finalmente, a contribuição individual das características das mulheres no risco de cesariana corrobora os resultados anteriores descritos. Ainda que não surpreendentemente a patologia tenha influenciado a decisão mais freqüente pela cesariana, independente de ano do parto, os excessos mais importantes contribuindo para um aumento em 2004 estiveram também relacionados com turno do dia, escolaridade elevada e, de forma menos importante, à maior freqüência ao pré-natal. Resultados apontando para uma contribuição importante de fatores claramente não-médicos sustentam a hipótese de um aumento temporal na flexibilidade das decisões obstétricas.

A utilização da medida de prevalência no estudo deve ser levada em conta tanto ao se interpretar as potenciais relações de associação entre exposição e desfecho, quanto suas implicações na leitura do RAP e conclusões relativas aos fatores contribuindo para um aumento na ocorrência de cesarianas em 2004. Entretanto, ainda que os dados tenham sido colhidos de maneira transversal, as características da relação temporal entre exposição e desfecho permite, em certo grau, a interpretação das diferenças nas proporções do desfecho como uma estimativa aproximada do risco de cesariana entre estas mulheres. Da mesma forma é importante ter em mente que a interpretação do RAP, ao se comparar os dois anos, assume que a relação entre as múltiplas variáveis influenciando o desfecho tenha se mantido constante, quanto à direção do risco, nos dois anos.

Alguns estudos no Brasil concluem que as altas taxas de cesariana refletem fatores sócioculturais da prática obstétrica, como a organização da prática médica, fatores institucionais, financeiros e legais, que constituem a "cultura da cesariana no Brasil” 31,32. Ainda apontam para uma cultura de uso abusivo de tecnologia médica no parto como a responsável por grande parte das cesarianas 9 .

Uma maior prevalência do procedimento entre mães de recém-nascidos prematuros nos dois anos estudados aponta para a ocorrência de "casualidade reversa", em que a utilização excessiva da cesariana como cirurgia eletiva estaria levando a maiores índices de prematuridade, e não vice-versa, fato também relatado em estudo semelhante conduzido no Rio Grande do Sul 15.

Medidas eficazes visando à redução de altas taxas de cesariana devem enfocar principalmente mudanças na prática obstétrica. A grande dificuldade em mudar conceitos já arraigados à sociedade médica foi relatada em recente estudo 32 . A hipótese do uso de segunda opinião para reduzir as taxas de cesariana sem comprometer indicadores maternos e perinatais foi testada em alguns hospitais de países latino-americanos com queda abaixo do esperado 33 . Os autores atribuíram esses resultados às dificuldades em mudar atitudes médicas. A alta concordância dos obstetras quanto às decisões (96\%) advogam esse pressuposto.

A compreensão da contribuição de fatores sócio-culturais no aumento das taxas de cesariana é crescente, mas ainda não há consenso sobre as melhores soluções para o problema. Países como os Estados Unidos e o Canadá implantaram medidas como a reestruturação do ensino médico e mudanças na forma de pagamento médico e hospitalar 33,34.

O Programa de Humanização do Pré-Natal e Nascimento do Ministério da Saúde, lançado em junho de 2000, fundamenta-se no direito à humanização da assistência obstétrica e neonatal como condição primeira para adequado acompanhamento, além de estabelecer critérios para qualificar a assistência e promover o vínculo 
entre a assistência ambulatorial e o momento do parto, no qual a efetividade tenha sido comprovada 35 . Entretanto, os resultados dessas medidas ainda não foram amplamente divulgados.

Intervenções eficazes, portanto, devem enfocar meios multidisciplinares, como a equipe médica, a organização dos serviços nas maternidades, além de se basear em conhecimento das particularidades sócio-culturais locais 15. Dessa forma, a informação deve chegar também até às mulheres, combatendo, assim, o estigma levando ao entendimento errôneo da cesariana como um "bem de consumo", ou como utilizada no sentido de favorecer preferencialmente àquelas mulheres com maior acesso sócio-econômico 12.

Uma conclusão importante a partir dos resultados é que a decisão quanto ao tipo do parto no HU-UFSC esteve relacionada não apenas a fatores médicos, mas sofreu influência importante de fatores não-médicos, tanto institucionais quanto da prática médica, apontando para uma provável maior permeabilidade do sistema público às práticas do sistema privado.

\section{Resumo}

O objetivo deste estudo foi descrever fatores associados ao aumento nas taxas de cesariana em um hospital universitário de 2002 e 2004, explorando a contribuição de fatores médicos e não-médicos para este aumento. Um estudo transversal investigou 2.905 partos ocorridos nos anos de 2002 (1.441) e 2004 (1.464). Diferenças nas razões de prevalência ajustadas pela regressão de Poisson e risco atribuível percentual foram estimados para a associação de cesariana com fatores sócio-demográficos; clínicos e reprodutivos; e do parto, institucionais e da prática obstétrica. As taxas de cesariana aumentaram de 28,4\% em 2002 para $36,7 \%$ em 2004. Escolaridade materna elevada, hora do parto, presença de patologia e maior freqüência ao pré-natal foram os fatores que representaram a contribuição mais expressiva para o excesso em 2004, quando comparado a 2002. O aumento nas taxas de cesariana ao se comparar os dois anos pode ser atribuído, pelo menos em parte, a um crescimento nas indicações relativas $e$ condições não-médicas.

Cesárea; Fatores de Risco; Risco Atribuível; Hospitais Universitários
Esses resultados ganham especial importância por ocorrerem em uma maternidade que busca seguir as recomendações da OMS no sentido da humanização da assistência ao parto, entre elas: o incentivo ao parto vaginal, ao aleitamento materno, ao alojamento conjunto, à presença de acompanhante e à redução do excessivo intervencionismo tecnológico no processo do parto 35 .

O aumento nas taxas de cesariana ocorrido no ano de 2004, em relação a 2002, esteve principalmente associado ao maior nível de escolaridade materna e à ocorrência do parto em período diurno, além de maior número de consultas pré-natal.

Por outro lado, uma diminuição na contribuição relativa dos nascimentos prematuros associados à cesariana, no período, fala a favor de características da prática obstétrica, inerentes a um hospital escola como o HU-UFSC, onde as cesáreas eletivas decididas como parte do prénatal não constituem prática freqüente.

\section{Colaboradores}

P. F. Freitas participou da concepção, planejamento, coordenação, análise, interpretação dos dados e na revisão crítica do conteúdo e elaboração do texto final. T. M. Sakae foi responsável pela coleta e entrada dos dados, participou na redação, análise e elaboração do texto final. M. E. M. L. P. Jacomino participou da revisão da literatura, colaborou na coleta e digitação dos dados e na redação final.

\section{Agradecimentos}

Conselho Nacional de Desenvolvimento Científico e Tecnológico. 


\section{Referências}

1. Rezende J. Obstetrícia. 8a Ed. Rio de Janeiro: Editora Guanabara Koogan; 1998.

2. Moraes MS, Goldenberg P. Cesarianas: um perfil epidêmico. Cad Saúde Pública 2001; 17:509-19.

3. Faúndes A, Cecatti JG. Which policy for cesarean section in Brazil? Analysis of thends and consequences. Health Policy Plan 1993; 8:33-42.

4. World Health Organization. Appropriate technology for birth. Lancet 1985; 2:436-7.

5. Barros F, Vaughan JP, Victora CG, Huttly S. Epidemic of caesarean sections in Brazil. Lancet 1991; 338:167-9.

6. Belizan JM, Althabe F, Barros FC, Alexander S. Rates and implications of caesarean sections in Latin America: ecological study. BMJ 1999; 319:397-400.

7. Departamento de Informática do SUS. Sistema de Informações de Nascidos Vivos. http://www. datasus.gov.br/catalogo/sinasc.htm (acessado em 26/Fev/2006).

8. Faúndes A, Cecatti JG. A operação cesariana no Brasil. Incidência, tendências, causas, conseqüências e propostas de ação. Cad Saúde Pública 1991; 7:150-73.

9. D'Orsi E, Chor D, Giffin K, Angulo-Tuesta A, Barbosa GP, Gama AS, et al. Fatores associados à realização de cesarianas e qualidade da atenção ao parto no Município do Rio de Janeiro. Rev Saúde Pública 2005; 39:646-54.

10. Perez GJG, Lopez MGV, Pivaral CC, Muñoz A, Jalle A. Cesarean sections in Mexico: are there too many? Health Policy Plan 2001; 16:62-7.

11. Diniz CSG. Entre técnica e os direitos humanos: possibilidades e limites da humanização da assistência ao parto [Tese de Doutorado]. São Paulo: Faculdade de Medicina, Universidade de São Paulo; 2001.

12. Behague DP, Victora CG, Barros FC. Consumer demand for caesarean sections in Brazil: informed decision making, patient choice, or social inequality? A population based birth cohort study linking ethnographic and epidemiological methods. BMJ 2002; 324:942-5.

13. Bailit JL, Love TE, Mercer B. Rising cesarean rates: are patients sicker? Am J Obstet Gynecol 2004; 191:800-3.

14. Joseph KS, Young DC, Dodds L, O'Connell CM, Allen VM, Chandra S, et al. Changes in maternal characteristics and obstetric practice and recent increases in primary cesarean delivery. Obstet Gynecol 2003; 102:791-800.

15. Freitas PF, Drachler ML, Leite JCC, Grassi PR. Desigualdade social nas taxas de cesariana em primíparas no Rio Grande do Sul. Rev Saúde Pública 2005; 39:761-7.

16. Yazlle MEHD, Rocha JSY, Mendes MC, Patta MC, Marcolin AC, Azevedo GD. Incidência de cesáreas segundo fonte de financiamento da assistência ao parto. Rev Saúde Pública 2001; 35:202-6.
17. Barros AJ, Hirakata VN. Alternatives for logistic regression in cross-sectional studies: an empirical comparison of models that directly estimate the prevalence ratio. BMC Med Res Methodol 2003; 3:21.

18. Szklo M, Nieto JF, editors. Epidemiology: beyond the basics. Gaithersburg: Aspen Publishers; 2000.

19. Gomes UA, Silva AAM, Bettiol H, Barbieri, MA. Risk Factors for the increasing caesarean section rate in Southeast Brazil: a comparison of two birth cohorts, 1978-1979 and 1994. Int J Epidemiol 1999; 28:687-94.

20. Lin HC, Sheen TC, Tang CH, Kao S. Association between maternal age and the likelihood of a cesarean section: a population-based multivariate logistic regression analysis. Acta Obstet Gynecol Scand 2004; 83:1178-83.

21. Lin HC, Xirasagar S. Maternal age and the likelihood of a maternal request for cesarean delivery: a 5-year population-based study. Am J Obstet Gynecol 2005; 192:848-55.

22. Bell JS, Campbell DM, Graham WJ, Penney GC, Ryan M, Hall MH. Do obstetric complications explain high caesarean section rates among women over 30 ? A retrospective analysis. BMJ 2001; 322:894-5.

23. Cabral SALCS, Costa CFF, Cabral Jr. SF. Correlação entre a idade maternal, paridade, gemelaridade, síndrome hipertensiva e ruptura prematura de membranas e a indicação de parto cesáreo. Rev Bras Ginec Obstet 2003; 25:739-44.

24. Uygur D, Gun O, Kelekci S, Ozturk A, Ugur M, Mungan T. Multiple repeat caesarean section: is it safe? Eur J Obstet Gynecol Reprod Biol 2005; 119:171-5.

25. Bais JMJ, van der Borden DMR, Pel M, Bonsel GJ, Eskes M, van der Slikke, et al. Vaginal birth after caesarean section in a population with a low overall caesarean section. Eur J Obstet Gynecol Reprod Biol 2001; 96:158-62.

26. Heffner LJ, Elkin H, Fretts RC. Impact of labor induction, gestational age, and maternal age on cesarean delivery rates. Obstet Gynecol 2003; 102:287-93.

27. Bailit JL, Dooley SL, Peaceman AN. Risk adjustment for interhospital comparison of primary cesarean rates. Obstet Gynecol 1999; 93:1025-30.

28. Bailit JL, Garret JM. Stability of risk-adjusted primary cesarean delivery rates over time. Am J Obstet Gynecol 2004; 190:395-400.

29. Patel RR, Peters TJ, Murphy DJ; ALSPAC Study Team. Prenatal risk factor for caesarean section. Analyses of the ALSPAC cohort of 12944 women in England. Int J Epidemiol 2005; 34:353-67.

30. Ehremberg HM, Durnwald CP, Catalano P, Mercer BM. The influence of obesity and diabetes on the risk of cesarean delivery. Am J Obstet Gynecol 2004; 191:969-74.

31. Freitas PF, Behague D. Brazil's culture of caesarean births. Health Exchange 1995; 10:8-9. 
32. Althabe F, Belizán JM, Villar J, Alexander S, Bergel E, Ramos S, et al. Mandatory second opinion to reduce rates of unnecessary caesarean sections in Latin America: a cluster randomised controlled trial. Lancet 2004; 363:1934-40.

33. MacFarlane A, Chamberlain G. What is happening to caesarean section rates? Lancet 1993; 342: 1005-6.
34. Millar WJ, Nair C, Wadhera S. Declining cesarean section rates: a continuing trend? Health statistics division at statistics Canada, Ottawa. Health Rep 1996; 8:7-24.

35. Tornquist CS. The paradoxes of humanized childbirth care in a public maternity ward in Brazil. Cad Saúde Pública 2003; 19:419-27.

Recebido em 13/Jul/2006

Versão final reapresentada em 03/Set/2007

Aprovado em 08/Out/2007 